\title{
ECONOMIES OF SCALE IN THE SWISS NURSING HOME INDUSTRY *
}

\author{
by \\ Massimo FILIPPINI \\ Department of Economics - Università della Svizzera Italiana \\ Via Ospedale 13 \\ 6900 Lugano, Switzerland \\ E-mail: massimo.filippini@lu.unisi.ch
}

January 1999

\begin{abstract}
This paper examines costs in the Swiss nursing home industry, an issue of concern to Swiss policy makers because of the explosion of elderly care costs and the aging of the population. The paper considers estimation of a translog cost function employing panel data for a sample of 36 nonprofit nursing homes operating in Ticino, a canton of Switzerland, over the period 1993-1995. Using a set of dummy variables quality differences are considered.
\end{abstract}

The results of this analysis indicate the existence of economies of scale for most output levels.

* Part of this work was done while I was a visiting research fellow at the Center for Health Economics, University of York (Summer 1998). I am grateful to Antonio Giuffrida and Andrew Jones for their comments on earlier versions of this paper and the Dipartimento Opere Sociali, Canton Ticino, for general assistance. The views expressed in this paper are strictly personal. Responsability for any remaining errors lies solely with the author. 


\section{Introduction}

The cost of residential provision for the elderly has risen steadily in the last 15 years. This dramatic increase and the negative situation of the Government's budget deficit have placed pressure on nursing homes to contain costs. A possible way to reduce costs per unit of service could be to promote nursing home mergers. Arguments for consolidation of the provision of residential services for the elderly are based on the presumed existence of economies of scale in the production of such service. If economies of scale exist, then a strong economic case could be made for the consolidation of small nursing homes in larger ones. Possible sources of such economies could be, for instance, a better utilization of the administrative, medical records and laundry services.

This paper estimates scale economies in the nursing home industry, using data from nursing homes operating in Ticino, a canton of Switzerland. ${ }^{1}$ For this purpose we estimate a translog cost function using data for 38 nonprofit nursing homes over the period 1993-1995. ${ }^{2}$ These nonprofit nursing homes are regulated in terms of quality standards and rates by the cantonal government, which gives them subsidies for the operating costs.

The paper is organized as follows. Section 2 discusses the cost model; section 3 presents the data, while section 4 illustrates the Parameter estimates of the total cost function and the results in terms of economies of scale. Section 5 concludes the paper.

\section{A Total Cost Function for the Nursing Homes}

The costs of operating a nursing home are the costs of the building and the equipment and the costs of taking care of the residents. A nursing home can, therefore, be represented as a firm transforming two inputs into patient-days of nursing home care. Moreover, in the cost model specification we take into account a number of output characteristic variables, which should capture the heterogeneity dimension of the output of a nursing home.

\footnotetext{
1 Switzerland is a confederation composed of 26 cantons and approximately 3000 municipalities. This federal state is characterized by a high degree of decentralization in the provision of public services. For instance, each canton is very independent in the organization and regulation of the provision of long-term care for elderly people. Moreover, some cantons decentralize this task to the communes (local government), while others tend to organize the provision of this service at the cantonal level.

2 See Vitalino \& al. (1994), Mobley and Bradford (1997) and McKeay (1990) for previous studies on the cost structure of Nursing Homes.
} 
Assuming that output and input prices are exogenous, and that (for a given technology) firms adjust input levels so as to minimize costs, the firm's total cost of operating a nursing home can be represented by the cost function

$\mathrm{TC}=\mathrm{h}\left(\mathrm{Y}, \mathrm{Q} 1, \mathrm{Q} 2, \mathrm{PK}_{\mathrm{K}}, \mathrm{PL}_{\mathrm{L}}, \mathrm{DIF}, \mathrm{T}\right)$

where $\mathrm{TC}$ represents total cost and $\mathrm{Y}$ is the output represented by the total number of patient-days of nursing home care. $\mathrm{PK}_{\mathrm{K}}$ and $\mathrm{PL}$ are the prices of capital and labor, respectively. Q1 is an index that measures the average required assistance of a home's patients with normal daily activities such as eating, personal care and performing physiological functions. This index is calculated yearly by the Regional Department of Public Health. Q2 is the ratio of the number of nurses employed by a nursing home and the number of nurses that should theoretically be employed according to the guidelines of the Regional Department of Public Health. ${ }^{3}$ These two variables, Q1 and Q2, are, therefore, introduced in the model as output characteristics. In order to distinguish nursing homes which offer the possibility for a relatively small part of the people to live with a small support in an apartment located beside the main building, we introduce in the model a dummy variable, DAPP. $T$ is a time variable which captures the shift in technology representing change in technical efficiency.

To be consistent with economic theory the translog cost function (2) should be concave and linearly homogeneous in input prices and non-decreasing in input prices and output. 4

To estimate the cost function (1), a translog functional form is employed. This flexible functional form is a local, second-order approximation to an arbitrary cost function. It places no a priori restrictions on the elasticities of substitution and allows the economies of scale estimate to vary with the output level..$^{5}$ The translog approximation to (1) is

\footnotetext{
3 The nursing homes are not obliged to exactly follow the guidelines of the regional Department of Public Health.

${ }^{4}$ See Cornes (1992), p.106.

5 A translog function requires the approximation of the underlying cost function to be made at a local point, which in our case is taken at the median point of all variables. Thus, all independent variables are normalized at their median point.
} 


$$
\begin{aligned}
\ln \left(\frac{T C}{P_{K}}\right)= & \alpha_{0}+\alpha_{y} \ln y+\alpha_{Q 1} \ln Q 1+\alpha_{Q 2} \ln Q 2+\alpha_{L} \ln \left(\frac{P_{L}}{P_{K}}\right)+\frac{1}{2} \alpha_{y y}(\ln y)^{2}+\frac{1}{2} \alpha_{Q 1 Q 1}(\ln Q 1)^{2} \\
& +\frac{1}{2} \alpha_{Q 2 Q 2}(\ln Q 2)^{2}+\frac{1}{2} \alpha_{L L}\left(\ln \frac{P_{L}}{P_{K}}\right)^{2}+\alpha_{y L} \ln y \ln \frac{P_{L}}{P_{K}}+\alpha_{y Q 1} \ln y \ln Q 1+\alpha_{y Q 2} \ln y \ln Q 2 \\
& +\alpha_{Q 2 Q 1} \ln Q 2 \ln Q 1+\alpha_{L Q 1} \ln \frac{P_{L}}{P_{K}} \ln Q 1+\alpha_{L Q 2} \ln \frac{P_{L}}{P_{K}} \ln Q 2+\alpha_{D A P P} D A P P+\alpha_{T} T
\end{aligned}
$$

Note that by normalizing total cost and input prices by one of the input prices, we impose the theoretical condition that the cost function is linearly homogeneous in input prices.

In order to improve the efficiency of the estimation of least squares parameter estimates for the cost function, a cost system is estimated. This system consists of the translog cost function (2) and the factor share equation for labor. By applying Shephard's lemma, the resulting share equation takes the familiar form:

$$
S_{L}=\alpha_{L}+\alpha_{L L} \ln \left(\frac{P_{L}}{P_{K}}\right)+\alpha_{y L} \ln y+\alpha_{L Q 1} \ln Q 1+\alpha_{L Q 2} \ln Q 2
$$

The price by which we normalize is that of the input whose share equation we dropped from the estimating system.

The translog cost function permits scale economies to vary with the level of output, factor prices and the output characteristic variables. Economies of scale (ES) are defined as the proportional increase in total cost resulting from a proportional increase in output (Y), holding all input prices and output characteristic variables fixed. This is equivalent to the inverse of the elasticity of total cost with respect to the output (Caves, Christensen, and Tretheway 1984) :

$$
E S=\frac{1}{\frac{\partial \ln T C}{\partial \ln Y}}=\frac{1}{\alpha_{y}+\alpha_{y y} \ln y+\alpha_{y L} \ln \frac{P_{L}}{P_{K}}+\alpha_{y Q_{1}} \ln Q 1+\alpha_{y Q_{2}} \ln Q 2}
$$

We will talk of economies of scale if ES is greater than 1, and accordingly, identify diseconomies of scale if ES is below 1 . In the case of ES $=1$ no economies or diseconomies of scale exist. Economies of scale exist if the average costs of a nursing home decrease as output increases. 


\section{The data}

This study is based on a combined time series and cross-sectional data set for 38 nonprofit nursing homes for elderly people operating over the period 1993-1995 in Canton Ticino.

For regulatory purposes each year the nonprofit nursing homes are required to send to the cantonal Department of Public Health information on operating costs and on the characteristics of the residents. ${ }^{6}$ Thus, the data set used in this study has been created using the information contained in these annual reports by the nonprofit nursing homes.

Total cost is taken to be the total expenditures of the nursing homes. Output is measured in total number of patient-days of nursing home care. Average yearly wage rates are estimated as the weighted mean of the average wage rates of the different professional categories working in a nursing homes: doctors, nurses, administrative and technical staffs.

Following Friedlaender and Wang Chiang (1983) and Filippini and Maggi (1993), the capital price is calculated from the residual capital costs divided by the capital stock. Residual cost is total cost minus labor cost. According to Wagstaffs (1989), the capital stock is approximated by the number of beds owned and operated by a nursing homes. Unfortunately no data are available which would allow us to calculate the capital stock using the capital inventory method. The quality indicators, Q1 and Q2, are calculated yearly by the regional Department of Public Health. All input prices, total costs and variable costs were deflated to 1996 constant Swiss francs using the Consumer Price Index.

\section{Estimation Results and Economies of Scale}

The multivariate system of equations (2) and (3) has been iteratively estimated using the Zellner (1962) procedure for seemingly unrelated regressions. ${ }^{7}$

The estimated coefficients of the translog cost model (2) are presented in Table 1. The results are satisfying in so far as all first order coefficients and most of the second order coefficients are significant and carry the expected signs. As can be seen, the corrected $\mathrm{R}^{2}$ for the model is also satisfying with a value of 0.98 . Since variable cost as well as the dependent variables are in natural logarithms and have been normalized, the first order coefficients are interpretable as cost elasticities evaluated at the sample median. The output elasticity is

\footnotetext{
${ }^{6}$ Unfortunately no data are available for the for-profit nursing homes. These nursing homes, which do not receive subsidies from the Department, are not obliged to send to the cantonal Department of Public Health information on operating costs and on the characteristics of the residents.

7 The model has been estimated using the computer program LIMDEP 7.0.
} 
positive and implies that a $1 \%$ increase in the number of patient-days of nursing home care will increase the total cost by approximately $0.86 \%$.

The cost elasticities with respect to the output chartacteristics variables, Q1 and Q2, are positive and imply that an increase in the average required assistance of a home's patients or an increase in the ratio of the number of nurses employed by a nursing home and the number of nurses that should be theoretically be employed will increase total cost. The estimated coefficient for time (proxy for technical change) is negative and significant, showing the presence of neutral technical change.

Table 1 : Total cost parameter estimates (standard errors in paranthese)

\begin{tabular}{|c|c|}
\hline Parameters & \\
\hline Constant & $\begin{array}{c}15.029 * * * \\
(0.017)\end{array}$ \\
\hline$\alpha_{Y}$ & $\begin{array}{c}0.860 * * * \\
(0.023)\end{array}$ \\
\hline$\alpha Q 1$ & $\begin{array}{c}0.844 * * * \\
(0.090)\end{array}$ \\
\hline$\alpha Q^{2}$ & $\begin{array}{c}0.630 * * * \\
(0.047)\end{array}$ \\
\hline$\alpha_{P L}$ & $\begin{array}{c}0.817 * * * \\
(0.002)\end{array}$ \\
\hline$\alpha Y Y$ & $\begin{array}{c}0.251 * * \\
(0.069)\end{array}$ \\
\hline$\alpha Q 1 Q 1$ & $\begin{array}{c}0.044 \\
(0.716)\end{array}$ \\
\hline$\alpha Q^{2} Q^{2}$ & $\begin{array}{c}0.504 * * \\
(0.122)\end{array}$ \\
\hline$\alpha P L P L$ & $\begin{array}{c}0.101 * * * \\
(0.545)\end{array}$ \\
\hline$\alpha_{Y Q 1}$ & $\begin{array}{c}0.816 \\
(0.225)\end{array}$ \\
\hline$\alpha Y Q 2$ & $\begin{array}{c}-0.408 * * \\
(0.122)\end{array}$ \\
\hline$\alpha Y P L$ & $\begin{array}{c}0.007 \\
(0.006)\end{array}$ \\
\hline$\alpha Q 2 P L$ & $\begin{array}{c}0.082 * * * \\
(0.008)\end{array}$ \\
\hline$\alpha Q 1 P L$ & $\begin{array}{c}0.186 * * * \\
(0.021)\end{array}$ \\
\hline$\alpha Q 1 Q 2$ & $\begin{array}{l}-0.009 \\
(0.244) \\
\end{array}$ \\
\hline$\alpha_{A P P}$ & $\begin{array}{c}0.297 \\
(0.017)\end{array}$ \\
\hline$\alpha_{T}$ & $\begin{array}{c}-0.013 * * \\
(0.006) \\
\end{array}$ \\
\hline $\mathrm{R}^{2}$ & 0.986 \\
\hline
\end{tabular}

*,**,*** significantly different from zero at the 90,95 and $99 \%$ confidence level. 
Parameter estimates of the cost function satisfy the regularity condition of concavity in input prices at the median point of approximation, which requires that the own-price elasticities of inputs be negative and that the Hessian Matrix, $\left[\partial^{2} \mathrm{C} / \partial \mathrm{w}_{\mathrm{i}} \partial \mathrm{w}_{\mathrm{j}}\right]$, be negative semi-definite. Because homogeneity in input prices and symmetry of the second order terms were imposed, the estimated functions satisfy all regularity conditions of a theoretically valid total cost model.

\section{Economies of scale}

The estimation results from Table 1 can be utilized to calculate, using equation (3), the values of the economies of scale in the production of the Swiss nursing homes. Table 2 presents in more detail the results for small, medium-sized and large nursing homes, respectively. We note that all values of the indicator for economies of scale are greater than 1, which means that the majority of the nursing homes operate at an inappropriately low scale. Up to a capacity of about 120 beds, the economies of scale are exhausted.

Table 2 Economies of scale 8

\begin{tabular}{|c|c|c|c|}
\hline $\begin{array}{c}\text { Nursing } \\
\text { home size }\end{array}$ & small & medium & large \\
& $\begin{array}{c}\text { Patient-days }=15160 \\
\text { Bed capacity }=41\end{array}$ & $\begin{array}{c}\text { Patient-days }= \\
20187 \\
\text { Bed capacity }=57\end{array}$ & $\begin{array}{c}\text { Patient-days }=26733 \\
\text { Bed capacity }=80\end{array}$ \\
\hline ES Model 1 & 1.19 & 1.18 & 1.15 \\
\hline
\end{tabular}

\section{Conclusion}

The paper consider estimation of a translog cost function for a sample of 36 Swiss nursing homes using panel data for the period 1993-1995.

8 Equation (5) has been evaluated at the input prices and output characterisctics variables, Q1 and Q2, of the median nursing home. 
The empirical evidence indicates the existence of economies of scale for most output levels. This result suggests that efficiency gains could result from merging smaller nursing homes operating in the same area. Of course, mergers between two small nursing homes do not, as a rule, result in a single larger nursing homes. The merger will generate the sought after efficiencies only if the two nursing integrate some clinical and administrative functions so as to act as a single nursing home. Moreover, by planning the size of new nursing homes the economies of scale effects on the costs should be taken into account. However, the size of the nursing homes should not reach an overly large size which could determine a deterioration of the quality in term of human contacts.

\section{References}

Bradford W.D. and Mobley L.R. (1997) 'Behavioural differences among hospitals: it is ownership or location?', Applied economics, 29, 1125-1138.

Caves, W.C., Christensen, L.R. and Tretheway, M.W. (1984),Economies of Density versus Economies of scale:Why trunk and Local Service Airlaine Costs Differ, Rand Journal of Economics, 15, 471-489.

Cornes, R., (1992), Duality and Modern Economics. Cambridge University Press.

Filippini, M. and Maggi, R. (1993), Efficiency and Regulation in the Case of the Swiss Private Railways, Journal of Regulatory Economics, 5, 199-216.

Friedlaender, A.F. and Wang Chiang, S.J. (1983), 'Productivity Growth in the Regulated Trucking Industry', Research in Transportation and Economics, 1, pp. 149-184.

McKay N.L., (1990) 'An Econometric Analysis of Costs and Scale Economies in the Nursing Home Industry', Journal of Human Resources, 23, 57-75.

Wagstaff, A. (1989) "Estimating Efficiency in the Hospital Sector: a Comparison of Three Statistical Cost Frontier Models", Applied Economics, 21, pp. 659-672.

Vitalino D.F., Toren M.,(1994). Cost and efficiency in nursing homes: a stochastic frontier approach', Journal of Health economics, 13 , pp. 281-300.

Zellner, A., (1962), 'An Efficient Method of Estimating Seemingly Unrelated Regressions and Test for Aggregation Bias', Journal of the American Statistical Association, 58, 348-368. 